\title{
Scheme for automation and energy saving of the technological process for the production of functional beverages
}

\author{
Elena Mironova ${ }^{1, *}$, Elena Romanenko $^{1}$, Maria Selivanova ${ }^{1}$, Timur Aysanov ${ }^{1}$, and \\ Maria German ${ }^{1}$ \\ ${ }^{1}$ Stavropol State Agrarian University, lane Zootechnical 12, 355017, Stavropol, Russia
}

\begin{abstract}
The modern development of the food industry is accompanied by an increasing use of automated process control systems. The widespread use of automated control systems is caused by a significant economic effect, which is achieved by ensuring the specified qualities of the products produced regardless of subjective factors, reducing the loss of valuable products, reducing the labor intensity of production processes, and increasing the culture of production. The article presents a scheme of automation and energy saving of the technological process of production of functional beverages based on direct-pressed grape juice with increased nutritional and biological value due to the inclusion of feijoa and blackberry extracts in their composition. Monitoring of the technological process was carried out using a multi-channel stand. The main parameters of monitoring were the consumption of basic and auxiliary products; the temperature of pasteurization and cooling of direct-pressed juice and extracts from fruit and berry raw materials; the level of pulp and product in the tanks. The obtained experimental data and developed technological methods allowed us to offer an effective energy-saving technology for the production of high-quality functional beverages in the conditions of energy-saving management of the production process. To implement the proposed technology, a hardware and technological scheme for the production of functional beverages has been developed. Process monitoring allows you to reduce energy consumption by up to $30 \%$ by using modern automation and control tools.
\end{abstract}

\section{Introduction}

Both in Russia and in other countries, there is currently a steady trend of increasing consumer interest in food products enriched with natural biologically active substances, primarily of plant origin [1-3]. Today, consumers prefer natural products, including a positive attitude to functional products.

Drinks are the most technological products for creating new types of functional nutrition, while the most promising functional products are drinks based on natural juices, enriched with biologically active substances of plant origin. Fruit and vegetable juices,

\footnotetext{
* Corresponding author: elena_st_86@mail.ru
} 
which are the main component of soft drinks, contain vitamin C, beta-carotene, a complex of B vitamins, while the introduction of new ingredients in them is not very complicated [4]. Enriched with dietary fiber and trace elements, drinks can be used to prevent cardiovascular, gastrointestinal diseases, cancer and other diseases, as well as to eliminate various types of intoxication [5].

In connection with the said above, we have developed a technology for the production of functional beverages based on direct-pressed grape juices using promising varieties and natural fruit and berry raw materials [6], as well as a scheme for automation and energy saving of the technological process.

\section{Materials and methods}

The work was carried out on the basis of the training and research laboratory of technology of winemaking and food from vegetable raw materials of Stavropol State Agrarian University (Stavropol, Russia). The technological process was monitored using a multichannel stand.

\section{Results}

To automate the technological process, it is necessary to monitor it [7-9], the objects of which are the basic modes and parameters of beverage production - the consumption of main and auxiliary products; the temperature of pasteurization and cooling of direct-pressed juice and extracts from fruit and berry raw materials; the level of pulp and product in tanks.

To implement this process, a multi-channel stand was developed (Figure 1), placed on a mounting panel (size 600x600 mm), on which the TSX Premium programmable logic controller (PLC) of Schneider Electric and auxiliary equipment are installed. The stand consists of a basket (rack) on which the following modules are installed: the TSX PCY2600 power supply unit; the TSX p572623 controller with a processor and an integrated Ethernet ETY PORT module; the TSX DEY16d2 digital input module with 16 inputs; the TSX DSY08R5 digital output module with 8 outputs; the analog input module with 8 channels TSX AEY800; analog input module with 4 channels TSX AEY414; analog output module with 8 channels TSX ASY800. The stand also has 2 ABE RM2401 power supplies and 4 "Unison" electronic keys (providing a discrete output) for controlling 4 actuators. The stand is powered by a voltage of $220 \mathrm{~V} \sim 50 \mathrm{~Hz}$.

The stand provides 16 digital inputs and 8 digital outputs, as well as 12 analog inputs and 8 analog outputs. In the extreme case, the stand can be expanded to the following parameters: 1024 digital inputs and outputs and 80 analog inputs and outputs. The time for processing information by the PLC processor is 0.19-2.4 MS.

The Programmable logic controller (PLC) allows you to install the modules of input at a distance of up to $50 \mathrm{~m}$ and has a high speed of information exchange (over the internal busup to $10 \mathrm{Mbit} / \mathrm{s}$, response time to an external event $-1.1 \mathrm{~m} / \mathrm{s}$ ). The presence of electronic keys "Unison" in the auxiliary equipment allows you to control actuators with a voltage of 24-480 $\mathrm{V}$ and a current of up to $50 \mathrm{~A}$. The stand is equipped with a thermal protection machine Merlin Gerin. 


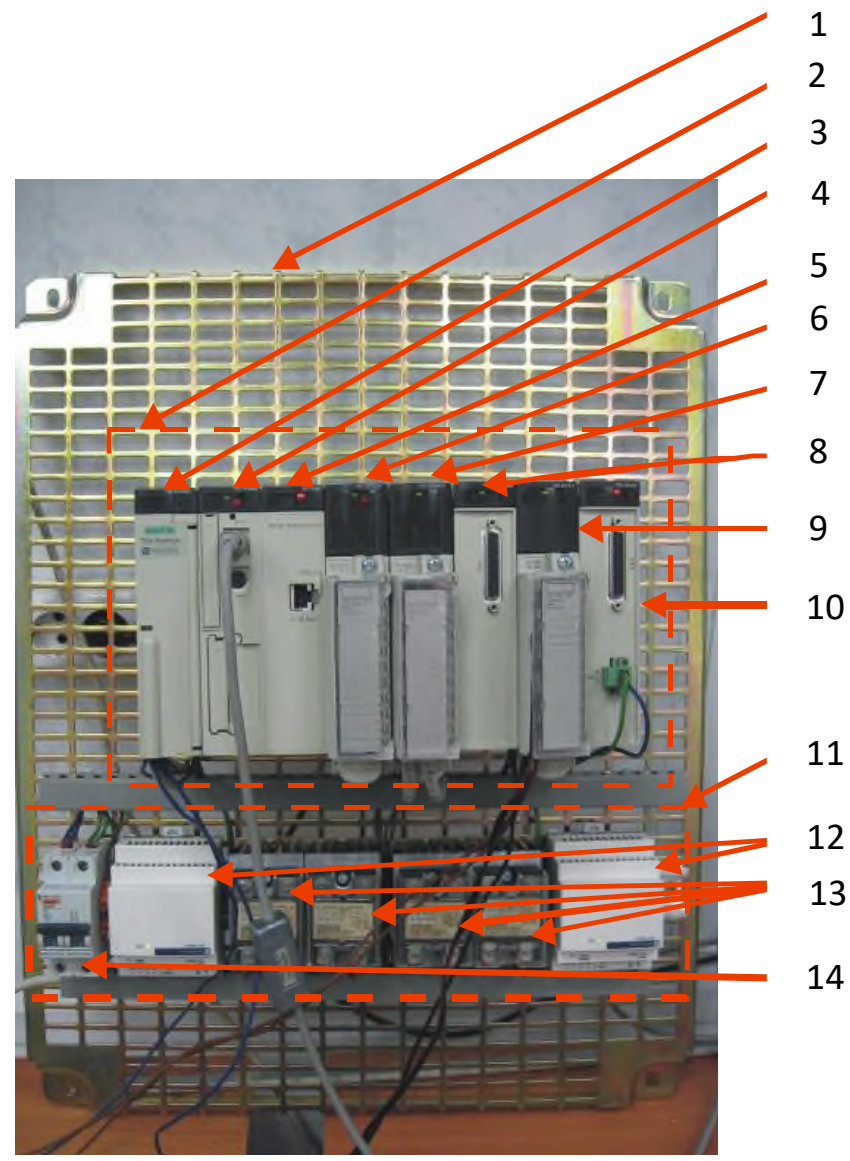

Fig. 1. Multichannel laboratory stand based on TSX Premium PLC: 1 - mounting panel; 2 programmable logic controller; 3 - the TSX PCY2600 power supply unit; 4, 5 - the TSX p572623 controller with a processor and an integrated Ethernet ETY PORT module; 6 - the TSX DEY16d2 digital input module with 16 inputs; 7 - the TSX DSY08R5 digital output module with 8 outputs; 8 the analog input module with 8 channels TSX AEY800; 9 - analog input module with 4 channels TSX AEY414; 10 - analog output module with 8 channels TSX ASY800; 11 - auxiliary equipment; 12 - power supply; 13 - electronic key; 14 - thermal protection machine.

To program and control the stand based on the TSX Premium PLC, you can use: Unity Pro software, PL-7; SCADA systems: Vijeo Citect, Monitor Pro.

For energy-saving control of three-phase asynchronous motors with a short-circuited rotor, a frequency-controlled electric drive based on Altivar frequency converters can be used [10].

The stand can be programmed from a workstation or laptop in the following ways: via a terminal port over the Uni-Telway bus; using networks: Ethernet, TCP/IP, Fipway, Modbus Plus (Figure 2). 


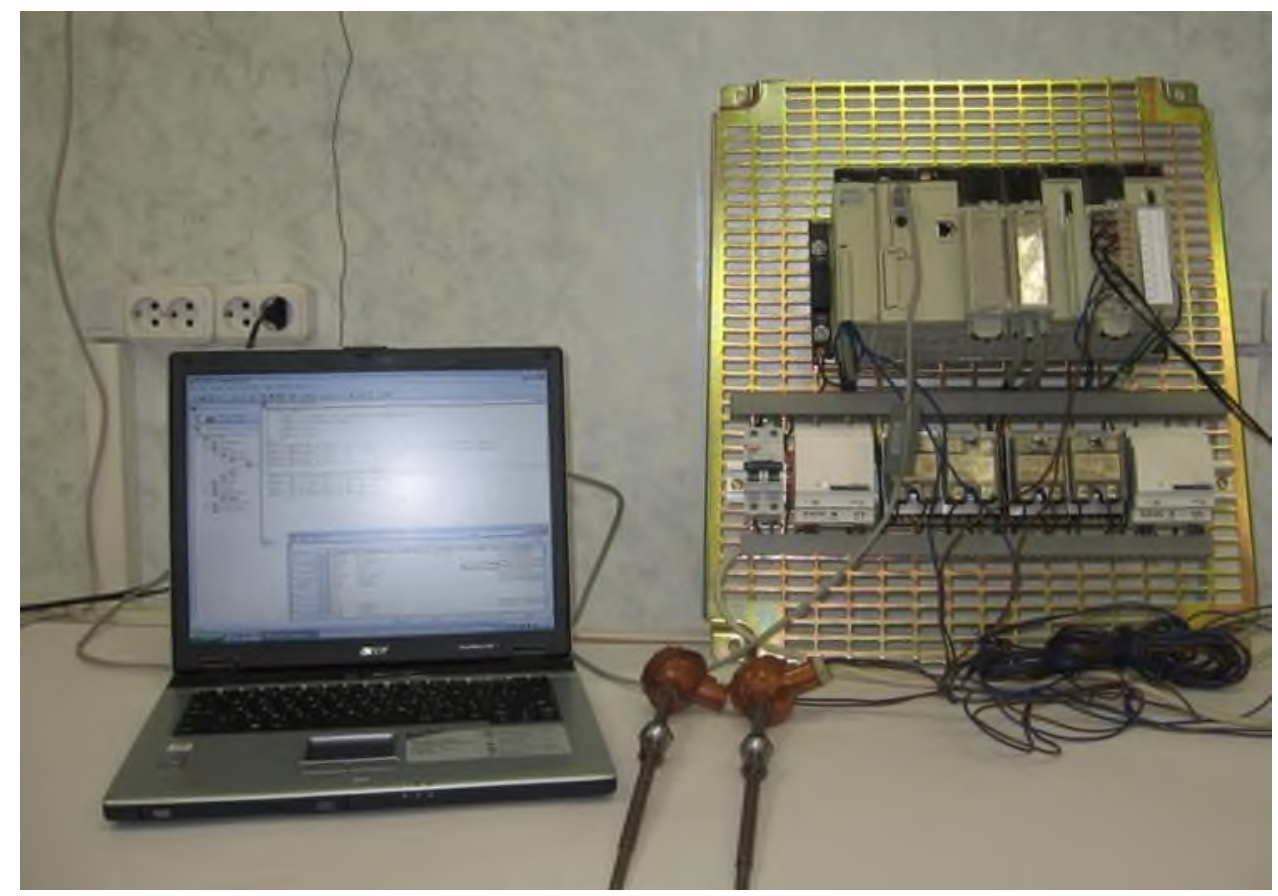

Fig. 2. Programming of a multichannel laboratory stand based on the TSX Premium PLC.

To confirm the reliability of the results obtained, statistical analysis was performed using mathematical methods (Fisher and student criteria) and the MAPLE 6 software package $[11,12]$.

The obtained experimental data and developed technological techniques allowed us to offer an effective energy-saving technology for the production of high- quality functional drinks in the conditions of energy-saving control of the production process [13]. To implement the proposed technology, a hardware and technological scheme have been developed (Figure 3) for the production of functional beverages based on direct-pressed grape juice with increased nutritional and biological value due to the inclusion of feijoa and blackberry extracts in their composition.

The grapes are fed into the feeder hopper (1), where the lower level sensor and the upper level sensor are installed. Next, the grapes are fed to the roller type crusher (2), in which a three-phase asynchronous motor (TAS) with a short-circuited rotor is installed on the frequency Converter ALTIVAR 58 with a power of $20 \%$ more than the power of the crusher motor. From the crusher (2), the comb conveyor (3) is used to remove the combs for disposal. In the comb conveyor (3), an ALTIVAR 31 frequency Converter with 20\% more power than the conveyor motor is installed on the TAS motor of the conveyor.

The resulting pulp from the crusher (2) is fed to a pneumatic drum-type press (5) by means of a pulp pump (4). The TAS pump motor (4) is equipped with an ALTIVAR 31 frequency Converter with $20 \%$ more power than the pump motor, and the TAS press motor (5) is equipped with an ALTIVAR 58 frequency Converter with $20 \%$ more power than the pneumatic press motor.

As a result of pressing in a pneumatic press (5), the must-gravity and the must of the press fractions are selected in the corresponding collections $(7,8)$. In order to automate the technological process, each of the collections $(7,8)$ has a lower level sensor and an upper level sensor installed. Then from the collection (8) using a centrifugal pump (13), the mustgravity is fed to the tank with a stirrer (9). The TAS motor of the pump (13) is equipped 
with an Altivar 31 frequency Converter with $20 \%$ more power than the pump motor, and a flow meter is installed at the pump outlet (13). A temperature sensor, as well as a lower level sensor and an upper level sensor are installed in the tank with the agitator (9). The ALTIVAR 31 frequency Converter with $20 \%$ more power than the agitator motor is installed on the TAS agitator motor $[14,15]$.

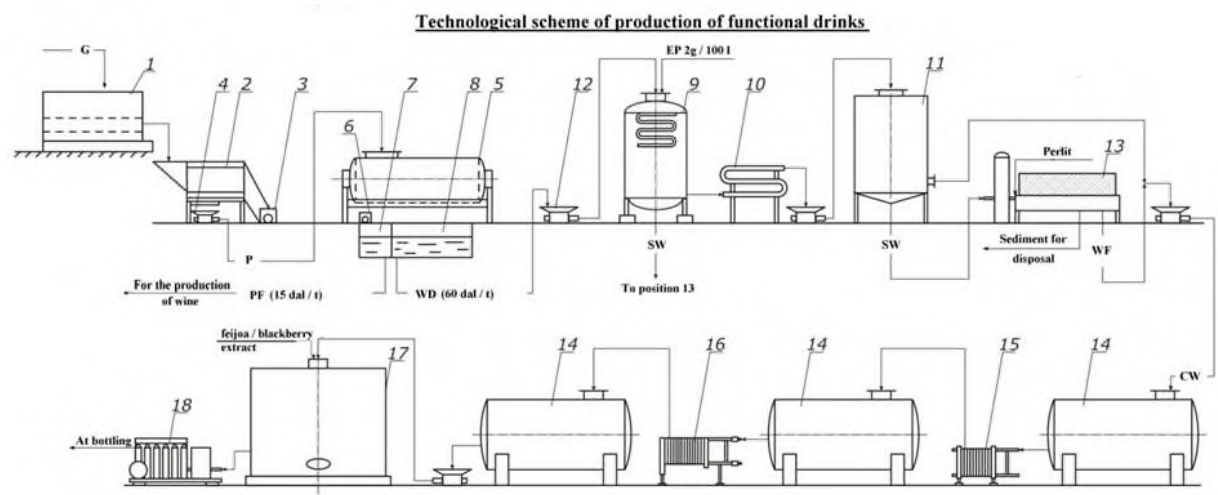

Technological scheme of production of feiioa and blackberrv extract

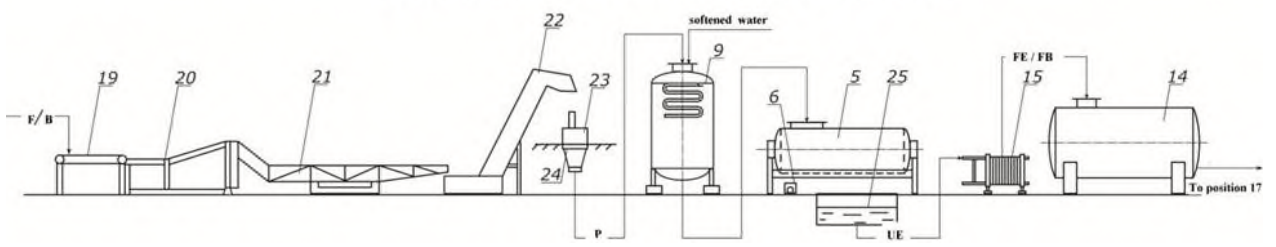

Fig. 3. Technological scheme of production of functional drinks based on grape juice: 1 - feed hopper; 2 - crusher; 3 - conveyor; 4 - pump for pulp; 5 - pneumatic press; 6 - conveyor for pomace; 7 - sump for pressing fractions (PF) ; 8 - sump for wort-drift (WD); 9 - tank with agitator; 10 cooler;11 - sump; 12 - pump; 13 - vacuum-perlite filter; 14 - tank; 15 - pasteurizer; 16 - ultracooler; 17 -blender; 18 - tangential membrane filter; 19 - roller con-veyor; 20 - washer; 21 - sorting and inspection conveyor; 22 - elevator; 23 - crusher; 24 - conical tank; 25 - tank for extract; $\mathrm{G}$ grapes; P - pulp; WD - wort-drift; PF - pressing fraction of wort; SW - sediment from wort, WF wort filtrate; CW - clarified wort; F - feijoa fruit; UE - unpasteurized extract; B - blackberries; FE feijoa extract; BE- blackberry extract.

In the tank (9), the wort is clarified using the pectolytic enzyme preparation in the dosage recommended by the manufacturer, at the rate of $2 \mathrm{~g} / 1001$ of wort, stirred and left alone for $1-2$ hours at a temperature of $18-20^{\circ} \mathrm{C}$.

Then the wort is cooled to a temperature of $4-6^{\circ} \mathrm{C}$ in a pipe-in-pipe cooler (10). For the purpose of automation, a temperature sensor and a flow sensor are installed in the cooler (10). The cooled wort from the cooler (10) is sent for clarification to the sump (11) for 1824 hours.

A temperature sensor, as well as a lower level sensor and an upper level sensor are installed in the sump (11); a flow meter is installed at the outlet of the sump.

After the specified settling time has elapsed, the clarified wort from the settling tank (11) is removed from the sediment, and with the help of a pump (13) it is sent for filtration through a vacuum perlite filter (15). Then, with the help of a pump (13), the clarified wort, after filtration on a vacuum perlite filter (15), is sent to a horizontal tank (16). At the outlet of the perlite filter (15), a flow meter is installed after the pump (13). A lower level sensor and an upper level sensor are installed in the horizontal tank (16). From a horizontal tank, the wort is fed to the pasteurizer for pasteurization (17). Pasteurization is carried out at a temperature of $82-85^{\circ} \mathrm{C}$ for $2-2.5$ minutes. The temperature sensor with a scale from 0 to 
$100{ }^{\circ} \mathrm{C}$ is installed in the pasteurizer (17), and a frequency Converter ALTIVAR 31 is installed on the TAS motor of the pasteurizer with a power of $20 \%$ more than the power of the pasteurizer motor.

After pasteurization, the juice materials are cooled to minus $2{ }^{\circ} \mathrm{C}$ in an ultra-cooler (35) and sent for storage. The ultra-cooler (35) is equipped with a temperature sensor with a scale from minus $10^{\circ} \mathrm{C}$ to plus $10^{\circ} \mathrm{C}$, and the TAS pasteurizer motor is equipped with the Altivar 31 frequency Converter with $20 \%$ more power than the pasteurizer motor.

Before sending for bottling, the juice material is decanted from the precipitation, blended in a blend (18) with extracts of vegetable raw materials in accordance with the recipe and filtered. In the blend (18), a lower and upper level sensor are installed, as well as a metering pump for dispensing feijoa and blackberry extracts. The TAS agitator motor is equipped with the ALTIVAR 31 frequency Converter with a power of $20 \%$ more than the power of the blending agitator motor.

Filtration is performed using a membrane tangential filter (19). The TAS tangential filter motor is equipped with the ALTIVAR 31 frequency Converter with $20 \%$ more power than the membrane tangential filter motor (19).

The clarified blend of the functional drink is sent to the pressure tank (20). The pressure tank is equipped with a lower level sensor and an upper level sensor, and a flow sensor is installed at the outlet of the tank. The finished functional drink is sent to an automated filling line (21-27).

By analogy, the lines for preparing feijoa and blackberry extracts are automated.

\section{Discussion}

As a result of the research, a scheme for automating the technological process of production of functional beverages from direct-pressed grape juices using promising varieties and natural fruit and berry raw materials has been developed. At all stages of the production, the technological process is monitored, which reduces energy consumption by up to $30 \%$ due to the use of modern automation and control tools (frequency-controlled electric drive for energy-saving control of three -phase asynchronous motors based on Altivar converters).

\section{References}

1. M. Serafini, A. Stanzione \& S. Foddai, Functional foods: traditional use and European legislation, International, Journal of Food Sciences and Nutrition 63(1), 7-9 (2012) https://doi.org/10.3109/09637486.2011.637488

2. D. Sun-Waterhouse, The development of fruit-based functional foods targeting the health and wellness market: a review, International, Journal of Food Science \& Technology 46, 899-920 (2011) doi:10.1111/j.1365-2621.2010.02499.x

3. S. Rodino, M. Butu, Herbal Extracts - New Trends in Functional and Medicinal Beverages, Functional and Medicinal Beverages 11, 73-108 (2019) https://doi.org/10.1016/B978-0-12-816397-9.00003-0

4. A. N. M. Alamgir, Therapeutic Use of Medicinal Plants and Their Extracts 1 (2017) https://doi.org/10.1007/978-3-319-63862-1

5. V. K. Atanasova, P. D. Gatseva, Natural Beverages and Their Role as Functional Foods, Natural Beverages 13, 37-71 (2019) https://doi.org/10.1016/B978-0-12816689-5.00002-X. 
6. E. A. Sosyura, E. S. Romanenko, M. V. Selivanova, et.al., Functional drinks based on grape juice and fruit and berry raw materials, IOP Conf. Series: Earth and Environmental Science 315 (2019) https://doi:10.1088/1755-1315/315/2/022048

7. A. Schumacher, W. Sihn, Development of a Monitoring System for Implementation of Industrial Digitalization and Automation using 143 Key Performance Indicators, Procedia CIRP 93 (2020) https://doi.org/10.1016/j.procir.2020.03.012

8. A. A. Ambartsumian, D. L. Kazansky, Complex automation of technological processes with involving event model in feedback control scheme, IFAC Proceedings Volumes 41, 2 (2008) https://doi.org/10.3182/20080706-5-KR-1001.00004

9. R. Syed, S. Suriadi, M. Adams, W. Bandara, S. J.J. Leemans, et.al., Robotic Process Automation: Contemporary themes and challenges, Computers in Industry 115 (2020) https://doi.org/10.1016/j.compind.2019.103162

10. JrJ. Lyu, M.N. Chen, Automated visual inspection expert system for multivariate statistical process control chart, Expert Systems with Applications 36, 3 (2009) https://doi.org/10.1016/j.eswa.2008.06.047

11. W.J.C. Verhagen, P. Bermell-Garcia, R.E.C. van Dijk, R. Curran, A critical review of Knowledge-Based Engineering: An identification of research challenges, Advanced Engineering Informatics 26, 1 (2012) https://doi.org/10.1016/j.aei.2011.06.004

12. M.A. Bezerra, V.A. Lemos, D.M. de Oliveira, et.al., Automation of continuous flow analysis systems - a review, Microchemical Journal 155 (2020) https://doi.org/10.1016/j.microc.2020.104731

13. E. Mironova, E. Romanenko, O. Sycheva, M. Selivanova, N. Esaulko, Optimal parameters and modes of extraction of biologically active substances from natural fruit and berry raw materials, E3S Web of Conferences 203, 04009 (2020) https://doi.org/10.1051/e3sconf/202020304009

14. Y. Lu, X. Xu, L. Wang, Smart manufacturing process and system automation - A critical review of the standards and envisioned scenarios, Journal of Manufacturing Systems 56 (2020) https://doi.org/10.1016/j.jmsy.2020.06.010

15. J. Lindholm, K. Johansen, Is Design Automation a Feasible Tool for Improving Efficiency in Production Planning and Manufacturing Processes?, Procedia Manufacturing 25 (2018) https://doi.org/10.1016/j.promfg.2018.06.074 